\title{
Suture repair of umbilical hernia during caesarean section: a case-control study
}

\author{
D. C. Steinemann $\cdot$ P. Limani $\cdot$ N. Ochsenbein . \\ F. Krähenmann · P.-A. Clavien · R. Zimmermann · \\ D. Hahnloser
}

Received: 1 July 2012/Accepted: 23 March 2013/Published online: 31 March 2013

(C) Springer-Verlag France 2013

\begin{abstract}
Purpose The objective of this study was to investigate the additional burdens in terms of pain, prolongation of surgery and morbidity which is added to elective caesarean section if umbilical hernia suture repair is performed simultaneously. Secondly, patient's satisfaction and hernia recurrence rate were assessed.

Methods Consecutive women with symptomatic umbilical hernia undergoing internal or external suture repair during elective caesarean were included in this retrospective cohortcontrol study. Data on post-operative pain, duration of surgery and morbidity of a combined procedure were collected. These patients were matched 1:10 to women undergoing caesarean section only. Additionally, two subgroups were assessed separately: external and internal suture hernia repair. These subgroups were compared for patient's satisfaction, cosmesis, body image and recurrence rate.
\end{abstract}

D. C. Steinemann $(\bowtie) \cdot$ P. Limani · P.-A. Clavien ·

D. Hahnloser

Department of Visceral and Transplantation Surgery, University

Hospital Zurich, Raemistrasse 100, 8091 Zurich, Switzerland

e-mail: daniel.steinemann@gmx.ch

D. Hahnloser

e-mail: dieter.hahnloser@chuv.ch

D. C. Steinemann

Department of Surgery, Cantonal Hospital Baselland,

4101 Bruderholz, Switzerland

N. Ochsenbein · F. Krähenmann · R. Zimmermann Department of Obstetrics, University Hospital Zurich, Raemistrasse 100, 8091 Zurich, Switzerland

D. Hahnloser

Department of Visceral Surgery, Centre Hospitalier Universitaire Vaudois, rue du Bugnon 21, 1011 Lausanne, Switzerland
Results Fourteen patients with a mean age of 37 years were analysed. Internal suture repair $(n=7)$ prolonged caesarean section by $20 \min (p=0.001)$ and external suture repair $(n=7)$ by $34 \mathrm{~min}(p<0.0001)$. Suture repair did not increase morphine use $(0.38 \pm 0.2$ vs. $0.4 \pm 02 \mathrm{mg} / \mathrm{kg}$ body weight), had no procedure-related morbidity and prolonged hospitalization by 0.5 days $(p=0.01)$. At a median follow-up of 37 (5-125) months, two recurrences in each surgical technique, internal and external suture repair, occurred (28\%). Body image and cosmesis score showed a higher level of functioning in internal suture repair ( $p=0.02 ; p=0.04$ ).

Discussion Despite a high recurrence rate, internal suture repair of a symptomatic umbilical hernia during elective caesarean section should be offered to women if requested. No additional morbidity or scar is added to caesarean section. Internal repair is faster, and cosmetic results are better, additional skin or fascia dissection is avoided, and it seems to be as effective as an external approach. Yet, women must be informed on the high recurrence rate.

Keywords Umbilical hernia - Caesarean section · Suture repair

\section{Introduction}

After inguinal and femoral hernias, umbilical hernias are the third most common type of hernia in women. Pregnancy represents a significant aetiological factor in the development of umbilical hernia [1], but the prevalence of umbilical hernia during pregnancy is largely unknown [2] and probably underestimated. Widening of the linea alba during pregnancy leading to a diastasis recti abdominis evokes pull forces on the umbilical ring which might lead to a 
symptomatic umbilical hernia [3-5]. There is still a debate about the ideal timing of hernia repair in pregnant women. Repair during pregnancy can prevent hernia complications such as incarceration with potential devastating outcome for mother and unborn child. However, umbilical hernias develop mostly in the second half of pregnancy when the umbilicus is covered by the growing uterus preventing hernia incarceration. Recent reports favour a "watchful waiting" strategy with post-partal hernia repair $[2,6]$. Although concerns are raised that hernia repair in the weakened and lax abdominal wall after pregnancy might not be promising, there is no evidence supporting these concerns in the current literature. Elective caesarean section gives a unique opportunity for simultaneous hernia repair in patients suffering from symptomatic hernia during pregnancy.

Three small case series have demonstrated the feasibility of combined caesarean section and umbilical or inguinal hernia repair with infection rates of $0-4.3 \%$ and recurrence rates of $0-2.1 \%$ [7-9]. However, inguinal and umbilical hernia repair were analysed together. Surgical technique of umbilical hernia repair was not standardized within the series and included open suture repair, open mesh-augmented repair and internal suture repair. The lack of homogeneous study groups makes the interpretation of these results difficult. In addition, hernia recurrence rates were not formally assessed by clinical and ultrasonographic examination. Therefore, recurrent umbilical hernia might have been missed. The question whether umbilical hernia repair should be performed during caesarean section and which approach should be chosen is still unanswered.

The objective of this study was therefore primarily to investigate the additional burden in terms of pain, prolongation of surgery and morbidity which is added to elective caesarean section if umbilical hernia suture repair is performed simultaneously. Secondly, cosmesis, patient satisfaction and recurrence rate after umbilical hernia repair for open suture repair and internal ring repair were assessed.

\section{Methods}

A retrospective study was performed in patients with simultaneous suture repair of a symptomatic umbilical hernia during elective caesarean section. Patients were identified in our electronic clinical information system between 2000 and 2011. Details of patient's age, body mass index, gravidity, parity, indication for the caesarean section, type of anaesthesia, duration of operation, loss in haemoglobin level, need for morphine, length of hospital stay and intra-hospital complications classified according to a validated grading system [10] were recorded.

In order to investigate operative morbidity as well as burdens by simultaneous umbilical hernia suture repair and caesarean section, the study group was case-matched to 140 consecutive caesarean sections without symptomatic umbilical hernia controlling for indication (elective first, second or third caesarean section of singletons or twins), parity and age ( \pm 1 year). The study and control groups were compared for duration of operation, post-operative pain analysed by morphine use in $\mathrm{mg} / \mathrm{kg}$ body weight, loss of haemoglobin and length of hospital stay.

For the assessment of cosmesis, patient's satisfaction and hernia recurrence, the study group was divided according to internal (IR) or external (ER) approach for the suture repair. Both subgroups, IR and ER, were compared. All patients were invited for a follow-up visit in our outpatient clinic. Patient's satisfactions with the procedure as well as with the cosmetic result were assessed by a visual analogue scale from 1 to 10 . Additionally, patients were asked to fill in a validated body image score (highest level of satisfaction $=20$ points, lowest level of satisfaction $=$ five points) and cosmesis score (highest level of satisfaction $=24$ points, lowest level of satisfaction $=3$ points) [11]. Hernia recurrence was evaluated clinically and controlled by ultrasonography in all patients.

Operative technique of external umbilical hernia repair (ER)

After closure of the Pfannenstiel incision of the caesarean section, a paraumbilical semilunar skin incision was performed. The hernia sack was dissected and opened. Afterwards, the hernia content was reduced. The fascia defect was closed either longitudinally or transversally using several interrupted sutures with non-absorbable material (polypropylene, 1-0, Ethicon Inc., Somerville, New Jersey, USA). Subcutis and skin were closed in the usual manner.

Operative technique of internal umbilical hernia repair (IR)

After closure of the uterotomy, the ventral abdominal wall was lifted upwards, allowing an exposure to the umbilical region. The hernia opening was identified by bimanual palpation on the outside and inside of the umbilicus. The fascia borders of the hernia were grasped with clamps, and several interrupted sutures were performed closing the fascia longitudinally using the same polypropylene suture material. Thereafter, the sutures were covered by duplicating the peritoneum with a running absorbable suture (Vicryl ${ }^{\circledR}$ 2-0, Ethicon Inc., Somerville, New Jersey, USA). This resulted in complete coverage of the polypropylene sutures with peritoneum avoiding any contact of the suture material to the bowel. The Pfannenstiel incision, subcutis 
Fig. 1 a The abdominal wall is lifted upwards, and the fascia borders of the umbilical hernia defect are palpated and grasped with Kocher clamps. b Several interrupted sutures using polypropylene are placed closing the fascia longitudinally. c After knotting of the sutures, the peritoneum is closed, thus covering the polypropylene sutures
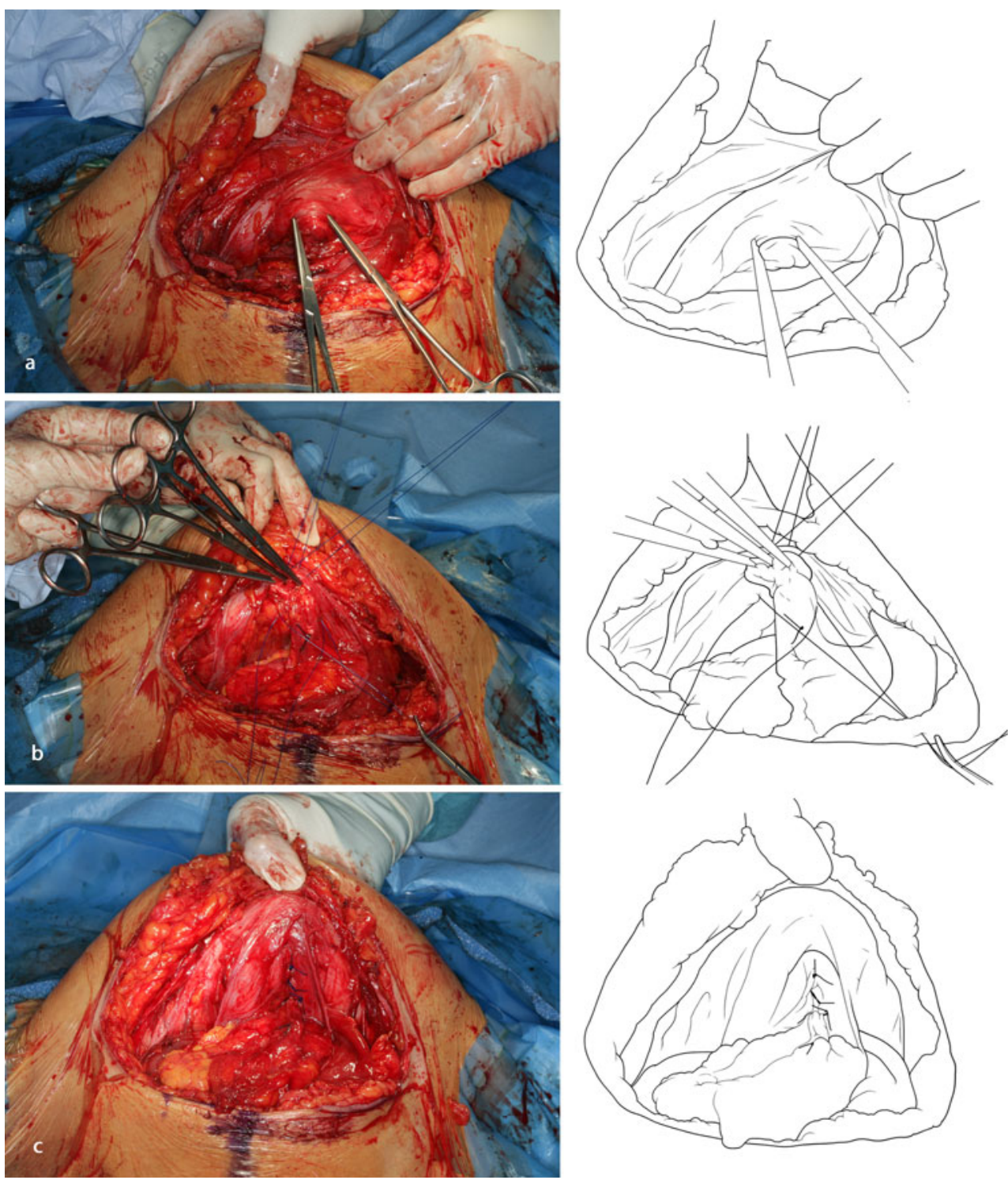

and skin were closed in the same standardized technique (Fig. 1).

\section{Statistical analysis}

Descriptive statistics and analysis of significant differences were performed using GraphPad $^{\circledR}$ Prism version 5.00 for Windows (GraphPad Software, San Diego California USA). For parameters expected to be normally distributed the mean and standard deviation and for parameter with a non-Gaussian distribution median and range are given. Proportions between groups were compared using a twotailed Mann-Witney test, paired and unpaired $t$ test where appropriate. Categorial variables were compared using a two-sided Fisher's exact test. The level of significance was set at 0.05 .

\section{Results}

During the study period, 14 patients with caesarean section combined with umbilical hernia repair were identified. These patients were not reported in our study of 2004 [9]. All patients presented with pain and tender protrusion being progressively during pregnancy and requested umbilical hernia repair. The mean age was 36.5 years $( \pm 4.0)$. Seven patients underwent open suture repair (ER) and seven patients internal ring repair (IR). All patients except for one in the ER group were operated in spinal anaesthesia. Six patients had a second caesarean section, four a third caesarean section and another four had an elective caesarean section for twins $(n=2)$ or breech presentation $(n=2)$. Patients in the study and the control group were well matched (Table 1). 
Table 1 Patient's characteristics
$S D$ standard deviation

\begin{tabular}{llll}
\hline & Study group $(n=14)$ & Control group $(n=140)$ & $p$ value \\
\hline Age (mean \pm SD) & $36.5 \pm 4.0$ & $37.4 \pm 2.8$ & 0.50 \\
Patients with/without first section & $2 / 12$ & $30 / 110$ & 0.74 \\
Patients with/without second section & $6 / 8$ & $60 / 80$ & 1.0 \\
Patients with/without third section & $4 / 10$ & $40 / 100$ & 1.0 \\
Patients with section for twins & $2 / 12$ & $10 / 130$ & 0.30 \\
Parity (median/range) & $3(2-4)$ & $2(2-4)$ & 0.30 \\
Gravidity (median/range) & $3(2-6)$ & $3(1-7)$ & 0.55 \\
Body mass index (mean \pm SD) & $22.9 \pm 4.2$ & $23.0 \pm 6.1$ & 0.64 \\
\hline
\end{tabular}

In-hospital outcome

The median diameter of umbilical hernia opening was 1.5 (range 1-5) cm. Hernia openings were closed longitudinally in all IR patients as well as in three ER patients and transversally in the remaining four ER patients. The mean duration of operation was significantly shorter in internal compared to external umbilical hernia repair (IR $54 \pm 11$ vs. ER $69 \pm 10 \mathrm{~min} ; p=0.04)$. However, compared to the control group in both subgroups, IR and ER, the duration of the operation was significantly prolonged (IR by $20 \mathrm{~min}, p=0.001$; and ER by $34 \mathrm{~min}, p<0.0001)$. The length of hospital stay was 0.5 days shorter in the control group compared to the study group (6.5 vs. 6.0 days, $p=0.01)$. Post-operative use of morphine did not differ between the study and the control group $(0.38 \pm 0.2$ vs. $\quad 0.40 \pm 0.2 \mathrm{mg} / \mathrm{kg}$ body weight; $p=0.68)$. The need of morphine was similar in the IR $(0.35 \pm 0.4)$ and the ER subgroup $(0.4 \pm 0.3 ; p=0.79)$. The drop in haemoglobin concentration after the procedure was similar in both the study and the control group (Table 2).

Only one Clavien-Dindo grade 2 complication [10] was recorded in a patient of the IR group requiring pharmacological therapy for subinvolution uteri. This corresponds to a complication rate of $7.1 \%(1 / 14)$.

Follow-up

The median follow-up time was 10 (range 5-45) in the IR and 55 (range 35-125) months in the ER subgroup $(p=0.005)$. Two recurrences in the IR and two in the ER subgroup occurred and confirmed by ultrasonography corresponding to an overall recurrence rate of $28 \%(4 / 14)$. The median time of onset of hernia recurrence was 4 (1-33) months. All four patients with recurrence presented initially with a small hernia defect $(\leq 2 \mathrm{~cm})$.

There was a tendency for a higher procedure satisfaction in the IR subgroup (10/10 points vs. $6 / 10$ points, $p=0.087)$. Both the median body image and cosmesis
Table 2 Outcome of simultaneous caesarean section and umbilical hernia suture repair and caesarean section alone

* Significant differences

Table 3 Comparison of internal $(n=7)$ and external $(n=7)$ suture repair of umbilical hernia during caesarean

* Significant differences

\begin{tabular}{lllc}
\hline & $\begin{array}{l}\text { Study group } \\
(n=14)\end{array}$ & $\begin{array}{l}\text { Control group } \\
(n=140)\end{array}$ & $p$ value \\
\hline Duration of operation (mean min $\pm \mathrm{SD})$ & $62 \pm 13$ & $35 \pm 18$ & $<0.0001^{*}$ \\
Haemoglobin loss $(\mathrm{g} \%)($ mean $\pm \mathrm{SD})$ & $1.04 \pm 0.6$ & $1.00 \pm 1.2$ & 0.89 \\
Length of stay (median days, range) & $6.5(5-29)$ & $6.0(2-74)$ & $0.012^{*}$ \\
Morphine use $\mathrm{mg} / \mathrm{kg}$ body weight (mean $\pm \mathrm{SD})$ & $0.38 \pm 0.24$ & $0.4 \pm 0.2$ & 0.68
\end{tabular}

\begin{tabular}{llll}
\hline & $\begin{array}{l}\text { Internal repair } \\
(n=7)\end{array}$ & $\begin{array}{l}\text { External repair } \\
(n=7)\end{array}$ & $p$ value \\
\hline Duration of operation (mean $\pm \mathrm{SD})$ & $54 \pm 11$ & $69 \pm 10$ & $0.036^{*}$ \\
Length of stay (median, range) & $5(5-29)$ & $8(5-9)$ & 0.13 \\
Morphine use mg/kg body weight (mean $\pm \mathrm{SD})$ & $0.35 \pm 0.23$ & $0.40 \pm 0.26$ & 0.80 \\
Recurrence rate & $2 / 7(28 \%)$ & $2 / 7(28 \%)$ & 1.0 \\
Satisfaction with procedure & $10(1-10)$ & $6(1-10)$ & 0.08 \\
Satisfaction with cosmesis & $10(3-10)$ & $6(1-10)$ & 0.23 \\
Modified body image scale & $20(19-20)$ & $18(10-20)$ & $0.022^{*}$ \\
Cosmesis scale & $24(12-24)$ & $13(3-17)$ & $0.038^{*}$ \\
\hline
\end{tabular}


score showed a higher level of functioning in the IR group $(p=0.02 ; p=0.04)$ (Table 3).

\section{Discussion}

This retrospective clinical cohort-control study compares a small group of patients $(n=14)$ with simultaneous umbilical hernia suture repair during caesarean section to a matched cohort of caesarean section only. Internal suture repair prolonged operation by $20 \mathrm{~min}$ and hospitalization by 0.5 days, but saved time for rehospitalization for separate hernia repair and subsequent convalescence. No procedure-related morbidity occurred, and recurrence rate was $28 \%$.

Umbilical suture repair is generally performed through a periumbilical incision. However, during caesarean section after the newborn is delivered, the umbilicus can also be approached from the Pfannenstiel incision in order to repair the hernia without the need for an additional external skin incision. While external repair through a periumbilical incision was preferred in the first time of the study period until 2008, internal suture repair was the method of choice thereafter. Therefore, ER and IR exhibit different followup periods. The duration of the operation was significantly prolonged compared to the control group, which is consistent with the previously published series [7-9]. However, IR added only $20 \mathrm{~min}$ to the caesarean section, resulting in a mean operation time of $54 \mathrm{~min}$. This short prolongation of the procedure does not affect the early and intense mother-child contact which has been shown to be important for bonding [12], because elective caesarean sections in spinal anaesthesia allow the mother to hold her newborn in her arms right after delivery till the end of the procedure.

No differences in post-operative need of analgesia and loss in haemoglobin were found between the study and the control group. Moreover, no procedure-related post-operative complication and especially no wound infection occurred. This finding is in line with the results of the Danish National hernia register reporting a $4.1 \%$ rate of mostly wound-related complications in umbilical hernia repair [13]. The median length of stay of 6 days, which is common for elective caesarean section in Switzerland, was prolonged by 0.5 days. However, this prolongation is not clinically relevant. Hence, in this study umbilical hernia suture repair during caesarean section did attribute neither to additional post-operative pain nor to morbidity nor to relevant prolongation of hospital stay compared to caesarean section alone.

In contrast to previously published series, our recurrence rate of $28 \%$ appears to be high. Longitudinal closure of the hernia opening applied in all IR and three ER patients might be challenged. However, reported recurrence rates in suture repair of umbilical hernia in non-pregnant adults vary between 11 and $54 \%$ [14-17]. Even in small hernia defects below $3 \mathrm{~cm}$, recurrence rates after suture repair up to $27 \%$ have been reported [18]. Therefore, the high recurrence rate in this study is comparable to recurrence rates in non-pregnant adults treated with direct suture repair.

Additionally, in contrast to previous reports combining suture repair and caesarean section, this study consists of a homogeneous cohort of umbilical hernia repair only. Furthermore, follow-up was performed not only clinically but also by ultrasonography. Therefore, the recurrence rate of $28 \%$ can be considered as accurate.

The advisability of combined umbilical hernia suture repair and caesarean section might be challenged by the high recurrence rate. Moreover, it is unknown whether umbilical hernias being symptomatic during pregnancy will remain symptomatic after delivery. There is no literature on the natural course of umbilical hernia after delivery. Yet, many women request repair of symptomatic umbilical hernia at the time of caesarean section. Women fear that umbilical hernia might cause problems in a further pregnancy. Furthermore, if the umbilical hernia has to be operated after delivery, young mothers will be separated from their babies in a vulnerable period of time and might get into trouble organizing babysitting for the time of hospitalization as well as the time they are not allowed to lift after repair of their hernia. Internal hernia repair combined with caesarean section does add neither additional morbidity nor additional skin nor additional fascia incisions. In approximately $70 \%$ of patients, it is a successful procedure which saves 3 days of rehospitalization for separate hernia repair. Therefore, we advocate that internal suture repair is offered if hernia repair is requested. However, it is important to inform women on the high recurrence rate.

Although a tenfold reduction in hernia recurrence can be achieved by mesh reinforcement [16], there is little reported experience with simultaneous umbilical hernia mesh repair during caesarean section. Yet, the feasibility has been shown, and no infectious complications have been reported [7]. According to the experience in non-pregnant patients, the morbidity rate is not increased in mesh versus suture repair [13]. Insertion of intra-peritoneal onlay mesh during section could be worthwhile to evaluate within clinical studies.

Patient's satisfaction with combined umbilical hernia repair and caesarean section in this study was high. Comparing the two surgical techniques, there was a tendency for higher procedure satisfaction with internal compared to external repair. Patients who underwent internal repair were significantly happier with their body image and less 
bothered by their umbilical scar. In this study, only symptomatic patients (mainly pain and tender protrusion) were operated, and therefore, only conclusions for this population of women can be drawn. Of note, most of the study population underwent second or third caesarean section, were well matched, but cannot be compared to elective low-risk caesarean section.

In summary, despite a recurrence rate of $28 \%$, simultaneous umbilical suture repair during caesarean section is worth an attempt given the fact that no additional pain and morbidity are added and rehospitalization days for separate hernia repair are saved. However, women need to be well informed on the high recurrence rate before surgery. Mesh reinforcement should be evaluated in future studies.

\section{Conflict of interest None.}

\section{References}

1. Dabbas N, Adams K, Pearson K, Royle G (2011) Frequency of abdominal wall hernias: is classical teaching out of date? JRSM Short Rep 2(1):5

2. Buch KE, Tabrizian P, Divino CM (2008) Management of hernias in pregnancy. J Am Coll Surg 207(4):539-542

3. Boissonnault JS, Blaschak MJ (1988) Incidence of diastasis recti abdominis during the childbearing year. Phys Ther 68(7): 1082-1086

4. Oh CS, Won HS, Kwon CH, Chung IH (2008) Morphologic variations of the umbilical ring, umbilical ligaments and ligamentum teres hepatis. Yonsei Med J 49(6):1004-1007

5. Beischer NA, Wein P (1996) Linea alba pigmentation and umbilical deviation in nulliparous pregnancy: the ligamentum teres sign. Obstet Gynecol 87(2):254-256

6. Perry Z, Netz U, Yitzhak A, Mizrahi S (2011) Pros and cons in the approach to an incarcerated umbilical hernia in the pregnant woman. Am Surg 77(3):E43-E44
7. Ghnnam WM, Helal AS, Fawzy M, Ragab A, Shalaby H, Elrefaay E (2009) Paraumbilical hernia repair during cesarean delivery. Ann Saudi Med 29(2):115-118

8. Gabriele R, Conte M, Izzo L, Basso L (2010) Cesarean section and hernia repair: simultaneous approach. J Obstet Gynaecol Res 36(5):944-949

9. Ochsenbein-Kolble N, Demartines N, Ochsenbein-Imhof N, Zimmermann R (2004) Cesarean section and simultaneous hernia repair. Arch Surg 139(8):893-895

10. Dindo D, Demartines N, Clavien PA (2004) Classification of surgical complications: a new proposal with evaluation in a cohort of 6,336 patients and results of a survey. Ann Surg 240(2):205-213

11. Dunker MS, Stiggelbout AM, van Hogezand RA, Ringers J, Griffioen G, Bemelman WA (1998) Cosmesis and body image after laparoscopic-assisted and open ileocolic resection for Crohn's disease. Surg Endosc 12(11):1334-1340

12. Nolan A, Lawrence C (2009) A pilot study of a nursing intervention protocol to minimize maternal-infant separation after Cesarean birth. J Obstet Gynecol Neonatal Nurs 38(4):430-442

13. Bisgaard T, Kehlet H, Bay-Nielsen M, Iversen MG, Rosenberg J, Jorgensen LN (2011) A nationwide study on readmission, morbidity, and mortality after umbilical and epigastric hernia repair. Hernia 15(5):541-546

14. Arroyo A, Garcia P, Perez F, Andreu J, Candela F, Calpena R (2001) Randomized clinical trial comparing suture and mesh repair of umbilical hernia in adults. Br J Surg 88(10):1321-1323

15. Stabilini C, Stella M, Frascio M, De Salvo L, Fornaro R, Larghero G, Mandolfino F, Lazzara F, Gianetta E (2009) Mesh versus direct suture for the repair of umbilical and epigastric hernias. Ten-year experience. Ann Ital Chir 80(3):183-187

16. Aslani N, Brown CJ (2010) Does mesh offer an advantage over tissue in the open repair of umbilical hernias? A systematic review and meta-analysis. Hernia 14(5):455-462

17. Halm JA, Heisterkamp J, Veen HF, Weidema WF (2005) Longterm follow-up after umbilical hernia repair: are there risk factors for recurrence after simple and mesh repair? Hernia 9(4):334-337

18. Israelsson LA, Smedberg S, Montgomery A, Nordin P, Spangen L (2006) Incisional hernia repair in Sweden 2002. Hernia 10(3):258-261 